\title{
ESPACIO SOCIAL Y TERRITORIALIDAD DE SOCIEDADES PREHISPÁNICAS DEL CHACO HÚMEDO ARGENTINO
}

\author{
Social Space and Territoriality of Pre-Hispanic Societies of the Argentine \\ Humid Chaco
}

\section{Gabriel Balbarrey, Guillermo Lamenza, Mariano Santini Carlos De Feo, Susana Salceda y Horacio Calandra}

\begin{abstract}
Resumen
Las investigaciones arqueológicas realizadas en los últimos años en el Chaco Meridional han contribuido a esclarecer el panorama de los patrones de asentamiento, formas de producción, subsistencia, costumbres funerarias y otros aspectos organizacionales de los antiguos pobladores de la región. Los sitios arqueológicos en estudio se encuentran en las proximidades de la localidad de La Eduvigis, Departamento San Martín, Chaco, emplazados sobre albardones conformando sobreelevaciones monticulares de origen natural y antrópico. En ellos, se han recuperado abundantes restos cerámicos y óseos, pertenecientes a fauna terrestre y acuática, así como restos óseos humanos con particulares significaciones simbólicas. A partir del análisis de estos sitios se plantea un uso particular del espacio, con características culturales compartidas por los grupos que habitaron en este sector. Se discuten las evidencias arqueológicas a través de las cuales es posible dar cuenta de la conformación de un espacio social particular al norte de la confluencia ParaguayParaná. A partir del análisis de este espacio social construido planteamos la existencia de una territorialidad, conformada por un ámbito simbólico y material.
\end{abstract}

$<$ Arqueología $><$ Territorialidad $><$ Uso del espacio $>$

\begin{abstract}
Archeological research conducted in recent years in the Meridional Chaco have helped to clarify the settlement patterns, forms of production, livelihood, funeral customs and other organizational aspects of the region's ancient inhabitants. Archaeological sites under consideration are in the vicinity of La Eduvigis town, San Martín Department, Chaco Provinice, and they are located on 'albardones' shaped as raised mounds of natural and anthropic origin.

In those sites, we have recovered abundant remains of ceramics and bones of terrestrial and acquatic fauna, as well as human skeletal remains with particular symbolic meanings. After their analysis, we propose that these sites show a particular use of space, with cultural characteristics shared by the groups that inhabited the area. We discuss the archaeological evidence through which it is possible to account for the formation of a particular social space in the north of the confluence
\end{abstract}


of the rivers Paraguay-Paraná. From the analysis of this constructed social space we posit the notion of territoriality, composed by material and symbolic aspects.

$<$ Archaeology $><$ Territoriality $><$ Use of space $>$

\section{Introducción}

En los últimos años, las investigaciones arqueológicas en el sector ribereño Paraguay-Paraná han contribuido a esclarecer el panorama de los sistemas sociales prehispánicos (Calandra et al, 2000; Salceda et al, 2000; Santini et al, 2003; Desántolo et al, 2005; Lamenza et al, 2005; Lamenza et al, 2006; Santini y Plischuk, 2006; Lamenza et $a l$, 2007). En esta oportunidad nos proponemos realizar una contribución al conocimiento sobre el pasado de la región a partir de la presentación y discusión de las evidencias arqueológicas disponibles correspondientes al Holoceno tardío. A partir de examinar el registro arqueológico del sector norte de la confluencia de los ríos Paraguay-Paraná en relación al uso del espacio, al análisis de la variabilidad en la producción cerámica, entre otros, planteamos la presencia de posibles relaciones de interacción intra e inter regional entre los grupos que habitaron esta área y las vecinas, aún quedando por dilucidar el interrogante sobre la naturaleza de dichas interacciones.

\section{Territorialidad y espacio social}

Desde la aparición de nuestra especie, el hombre ha establecido su existencia transformando la naturaleza. El espacio natural, su entorno, fue transformado para convertirse en un espacio social. Por tanto, las distintas culturas tienen su propia forma de ver e interpretar el mundo, es por esto que el territorio es una parte más de la construcción social y su sistema de valores.

En el caso de los cazadores recolectores pescadores, y en nuestro estudio particular del sector ribereño, interpretamos al paisaje no como una simple acumulación de rasgos del relieve sino, siguiendo a Criado (1999), como una construcción en la que juegan tres esferas: el espacio físico (espacio natural sin intervención humana), el espacio social (medio construido por los individuos o grupos sociales) y el espacio simbólico (base de comprensión de la apropiación humana de la naturaleza).

Considerándolo desde un enfoque ecológico evolutivo, a partir de un análisis de costo/beneficio, observamos que cuando los recursos críticos son abundantes y predecibles en tiempo y espacio, es posible esperar una estrategia territorial (Dyson-Hudson y Smith, 1978). A su vez Cashdan (1983), en su aproximación al desarrollo de una teoría general de la territorialidad humana, plantea que las capacidades culturales y cognitivas del hombre han alterado las formas en que el territorio puede ser definido. Asimismo, es importante la percepción que el propio grupo tiene de su territorio y de los límites territoriales que mantiene con grupos vecinos (Barnard, 1983). En este sentido, el control al acceso de 
los recursos se puede dar ya sea por defensa activa del perímetro, o bien a través del establecimiento de pactos, acuerdos, normativas o tabúes restrictivos. Además, es posible que el tamaño del territorio disminuya a medida que los recursos son más abundantes y predecibles. (Cashdan, 1983).

\section{Características de los sitios}

Los sitios arqueológicos considerados en esta oportunidad se encuentran dentro de la Subregión de Esteros, Cañadas y Selvas de Ribera (Morello, 1968), al norte de la confluencia de los ríos Paraguay-Paraná (Figura 1). También es de particular importancia la información obtenida, en distintas oportunidades, a partir de la realización de prospecciones arqueológicas (De Feo et al, 2002).

Estos sitios se encuentran emplazados a la vera de cursos de agua secundarios del sistema Paraná-Paraguay ( $\mathrm{A}^{\mathrm{o}}$ Tucá, $\mathrm{A}^{\mathrm{o}}$ Quiá y $\mathrm{A}^{\mathrm{o}}$ Malá) y se disponen sobre líneas de albardón poco desarrolladas donde se instalan bosques riparios de inundación (Ginzburg y Adámoli, 2006). Se presentan como estructuras de ocupación que configuran montículos, sobreelevando el terreno por encima del nivel de inundación en la época de lluvia. Dichas estructuras comprenden recintos de grandes dimensiones, forma sub-oval (de aproximadamente $30 \mathrm{~m}$ de diámetro mayor), construidos con ramadas, tal como lo evidencian, entre otros indicadores, trozos de arcilla con improntas utilizados a manera de revoque. ${ }^{1}$

La matriz que contiene los materiales arqueológicos varía en cuanto a su potencia, oscilando entre los 0,30 y los $0,60 \mathrm{~m}$ de profundidad. Constituida, en todos los casos, por un sustrato compacto, húmedo, de color negro amarronado y de alto contenido orgánico. La mayor densidad de materiales (más de 500 fragmentos de cerámica y 300 restos de material óseo faunístico por $\mathrm{m}^{3}$ ) se encuentra dentro de una matriz de tierra conchífera (Piana, com pers) entre los 0,40 y $0,56 \mathrm{~m}$ de profundidad.

Los fechados radiocarbónicos disponibles se ubican en un rango cronológico acotado dentro del Holoceno tardío, tal como se muestra en la Tabla 1. Esta cronología, así como el análisis de la tecnología ósea (Santini y Plischuk, op. cit.) y del estilo cerámico (Lamenza et al, op. cit.) permiten entender a estos grupos como parte de un mismo sistema social con características culturales compartidas. A estas características se les suma una forma particular de organización en el espacio a través de la modificación activa del mismo con una funcionalidad que sin lugar a dudas trasciende el dominio del campo material. A su vez, esta modificación activa del espacio configura una significación que es parte de un modo de pensamiento donde trayectoria histórica (Pauketat, 2001) y práctica social no pueden dejar de ser tenidas en cuenta (Ingold 1986; 1999).

\footnotetext{
${ }^{1}$ Estas estructuras distribuidas espacialmente de a pares, por sus dimensiones y la disposición de los materiales recuperados, permiten avanzar en el análisis de la organización social y de la dimensión simbólica de estas poblaciones. Las primeras aproximaciones a estas temáticas serán presentadas en futuros trabajos.
} 


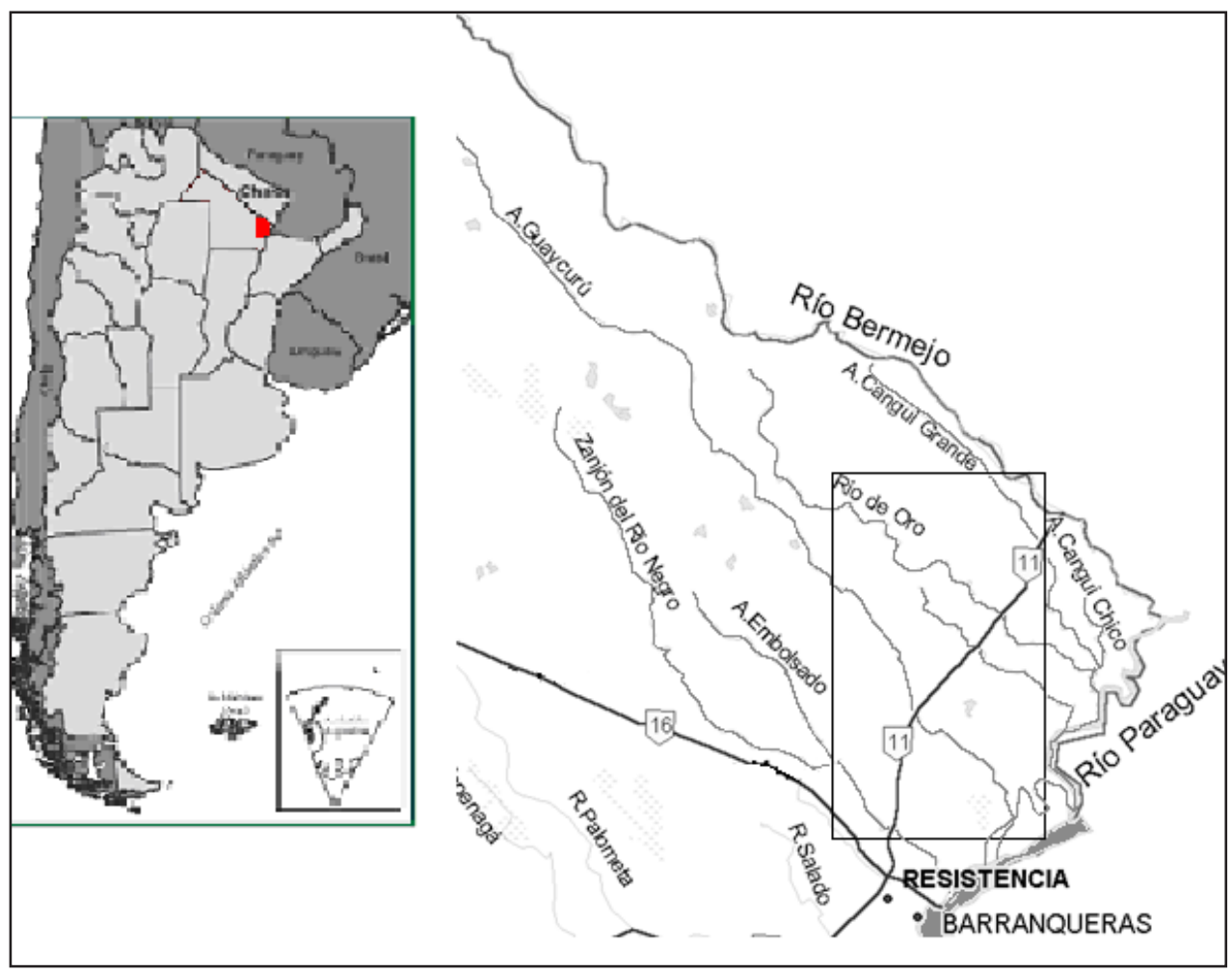

Figura 1. Mapa del área de estudio

\section{Habitando el montículo}

En todos los sitios se ha recuperado abundante material cerámico (más de 10000 fragmentos) representando diferentes etapas de la producción (manufactura, uso, reciclado y descarte). Este material se caracteriza por pastas con núcleos que van del gris al negro, con márgenes grisáceos y pardos, cocción oxidante incompleta, inclusiones de tiesto molido de tamaño mediano. La técnica de manufactura utilizada ha sido la de rodete. Los fragmentos poseen una dureza media ( 3 y 4 de la escala de Mohs) y su fractura es irregular. La matriz en general, presenta una textura media y porosa. Las formas presentes son abiertas no restringidas y restringidas, ambas de contornos simples, como son las escudillas, cuencos y ollas, con diámetros que oscilan entre $12-36 \mathrm{~cm}$. La decoración incluye incisiones de líneas finas y gruesas, de surco rítmico, punteados, presiones unguiculares y, con mayor frecuencia, corrugados, en sus variantes simple, inciso y unguiculado (fotos 1, 3, 4). En mayor medida se encuentra dispuesta en forma de guardas sobre el tercio superior de la pieza conformando motivos geométricos (foto 5). 
Tabla 1

Ubicación (GPS) y cronología expresada en años $\mathrm{C}^{14} \mathrm{AP}$ de los sitios arqueológicos en la región de estudio.

\begin{tabular}{|c|c|c|c|c|}
\hline Código & Nombre & $\begin{array}{c}\text { Ubicación } \\
\text { (GPS) }\end{array}$ & $\begin{array}{c}\text { Cronología (en años } \\
\text { radiocarbónicos C } \\
\text { AP) }\end{array}$ & Muestra \\
\hline SChaPrim4.1 & $\begin{array}{c}\text { El Cachapé } \\
- \text { Pot. V y } \\
\text { Anexo }\end{array}$ & $\begin{array}{c}-26,89 \mathrm{~S} \\
-59,01 \mathrm{~W}\end{array}$ & $920 \pm 60 \mathrm{AP}$ & P canaliculata \\
& El Cachapé - & $-26,87 \mathrm{~S}$ & $1180 \pm 70 \mathrm{AP}$ & B. dichotomus \\
\hline \multirow{2}{*}{ SchaPrim5.1 } & Pot. IVA & $-58,96 \mathrm{~W}$ & $1260 \pm 80 \mathrm{AP}$ & H. sapiens \\
\hline SchaPrim6.1 & $\begin{array}{c}\text { El Cachapé }- \\
\text { Pot. IVB }\end{array}$ & $\begin{array}{l}-26,86 \mathrm{~S} \\
-58,96 \mathrm{~W}\end{array}$ & $1680 \pm 100 \mathrm{AP}$ & B. dichotomus \\
\hline SchaSmar1.1 & Sotelo I & $\begin{array}{l}-26,79 \mathrm{~S} \\
-59,05 \mathrm{~W}\end{array}$ & $1690 \pm 90 \mathrm{AP}$ & M. coypus \\
\hline SchaSmar2.1 & Sotelo II & $\begin{array}{l}-26,78 \mathrm{~S} \\
-59,05 \mathrm{~W}\end{array}$ & - & - \\
\hline
\end{tabular}

Como mencionáramos en otra oportunidad (Lamenza et al, op. cit.), el análisis de la variabilidad en la producción cerámica presenta agrupamientos que reflejan la distribución espacial de los sitios, diferenciando un conjunto hacia el norte de la confluencia Paraguay-Paraná y otro hacia el sur de la misma. Dichos resultados nos permitieron aislar motivos decorativos que caracterizan y/o diferencian a los sitios. Por ejemplo, la cerámica incisa de surco rítmico y la punteada sobre el labio caracteriza a Puesto Fantín (al sur de la confluencia) en tanto que la cerámica corrugada, en sus distintas variantes, a los sitios El Cachapé Potrero IV-A, El Cachapé Potrero IV-B, El Cachapé Potrero V. Además, el corrugado, según la incidencia diferencial de cada una de sus variantes, explicaría la variabilidad dentro del subsector norte. En el fenograma de la figura 2 se visualiza esta relación.

Como propusiéramos en Lamenza et al (2006), y partiendo de considerar a la cerámica corrugada como un buen indicador, no sólo de dinámica social, sino también de relaciones particulares entre grupos, las similitudes y diferencias halladas nos permitieron plantear esta interacción. Específicamente, hacia el sur de la confluencia, en los sitios El Chancho (Colazo, 2002) y Puesto Fantin (Calandra et al, 2004) no se encuentra este motivo decorativo, lo cual no necesariamente indica ausencia de interacción, aunque sí plantea otro tipo de relaciones que no incluyen elementos ideológicos caracterizados por la significación de este estilo cerámico. En cambio hacia el norte, en el caso del Pantanal, encontramos estrechas similitudes tanto en las características ambientales, como en las características de los restos culturales (Schmitz et al, 1996). Así, Rogge (1996) dentro de la fase Pantanal describe la presencia de corrugado simple, corrugado simple 
riscado, corrugado simple digitado, entre otros, indicativos de interacción en función de su similitud con los estilos presentes en la región (véase fotos 1 y 2). Sin embargo, el análisis composicional de la cerámica en ambos sectores demuestra que su manufactura es local, hecho que permite considerar al corrugado como un estilo compartido más allá del intercambio o difusión de piezas cerámicas. Situación similar se ha planteado recientemente entre las tierras bajas ecuatorianas y el Pantanal utilizando los mismos indicadores (Arellano López, 2009). Lo expuesto, sumado a las características generales de los aterros (Schmitz y Beber, 1996) nos lleva a entender estas interacciones dentro de un mismo proceso histórico. Estos aterros se encuentran emplazados en relación con lagunas, pequeños ríos y campos anegadizos y presentan abundantes restos faunísticos, tanto acuáticos como terrestres.

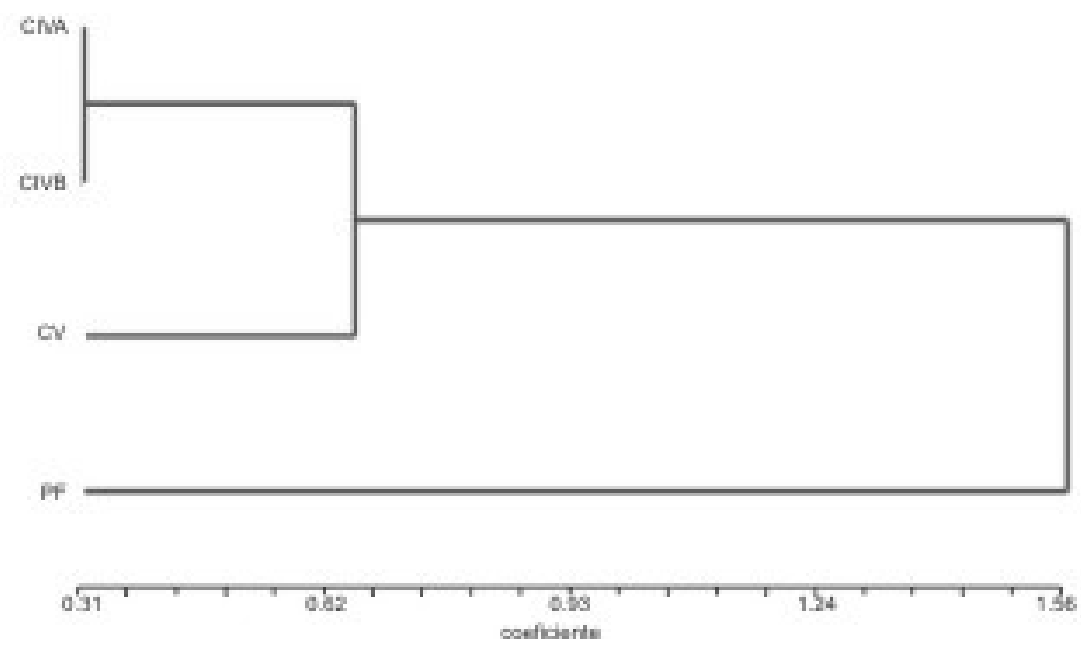

Figura 2. Análisis de clusters marcando agrupamientos entre los sitios del sector ribereño. $\mathrm{PF}=$ Puesto Fantin; $\mathrm{CV}=\mathrm{El}$ Cachapé Potrero V; CIVA= El Cachapé Potrero IVA; CIVB= El Cachapé Potrero IVB (Lamenza, et al 2005).

Hacia el oeste, dentro del sector central del Chaco argentino (Braunstein et al, 2002), Balbarrey et al (2003) y Calandra et al (2004) analizan material cerámico del subsector norte, encontrando, dentro de la cerámica corrugada, las variedades incisa, simple, unguiculada y complicada. Esta última variedad, sumada a la presencia de otros materiales (v.gr. filete aplicado), habilita la hipótesis de entender a esta producción cerámica como parte de un sistema social distinto al presente en El Cachapé. En el mismo sector, hacia el sudoeste de la provincia del Chaco, encontramos que las diferencias son aún más notables, hallándose mínimas evidencias que permitan vincular este material cerámico con el referido en esta oportunidad. (González, 2006 com. pers.). 
Folia Histórica del Nordeste, № 18 (Resistencia, 2010) IIGHI, CONICET - IH, UNNE

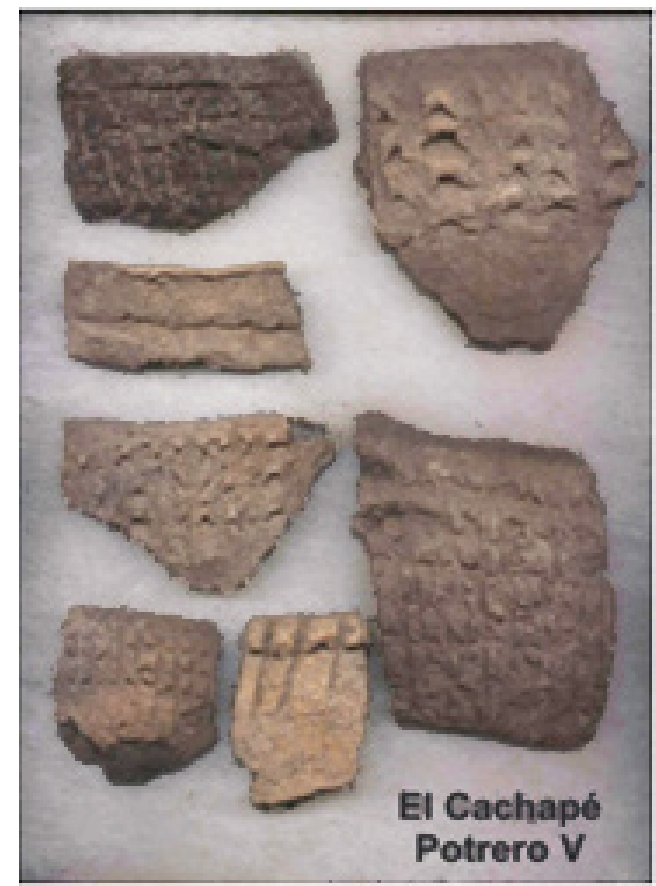

Foto . Cerámica con corrugado simple recuperado en PV

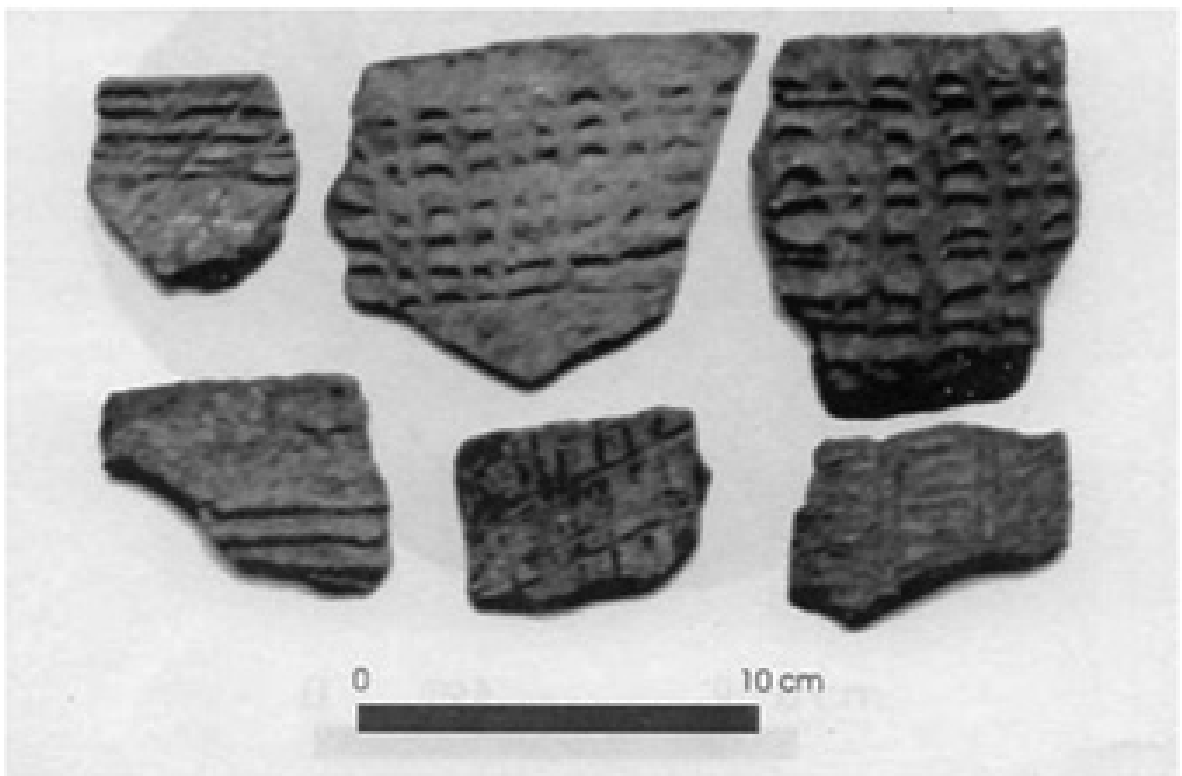

Foto 2. Cerámica con corrugado simple recuperado en el sitio 14 de Mayo, Paraguay (tomado de Eremites de Oliveira, 2004). 


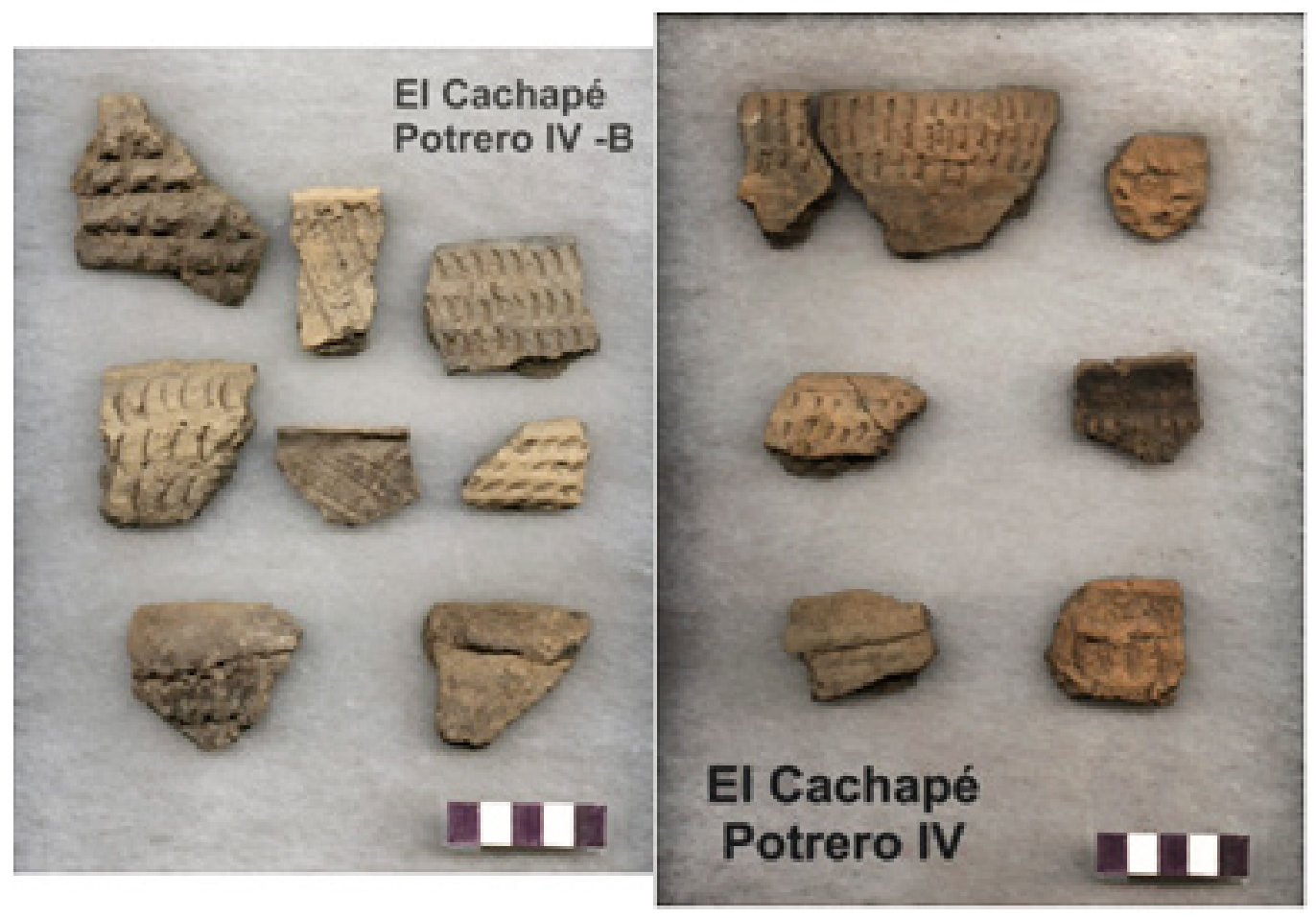

Fotos 3 y 4. Motivos decorativos presentes en los sitios de la región.

En la región que nos ocupa un recurso básico es el agua, como insumo crítico para la supervivencia y, no menos importante, como elemento aglutinante de recursos animales, vegetales y como sistema de comunicación. Su disponibilidad depende de los pulsos estacionales lluvia-seca y crecida-bajante, inundaciones extraordinarias-épocas de sequía (Neiff, 1986). No obstante el agua en la zona no deja de ser un recurso predecible y disponible, debido a los numerosos ríos que la atraviesan, los que alimentan a los también numerosos cuerpos de agua estables, esteros y bañados.

Los restos de fauna recuperados pertenecen en su gran mayoría a taxa de hábitos acuáticos (coipo, peces, aves, entre otros) con comportamientos altamente predecibles, homogéneamente distribuidos en el espacio y en el tiempo cuando se considera una escala anual. A su vez, son recursos multifuncionales ya que se aprovecharon tanto su carne, cueros y sus huesos fueron utilizados como materia prima para la confección de instrumentos (Santini, 2009), de fácil captura, procesamiento y susceptibles de almacenar.

Dentro de los restos faunísticos recuperados predominan los de Myocastor coypus (coipo) y Blastocerus dichotumus (ciervo de los pantanos) y como recursos complementarios, peces, moluscos, reptiles y Cavia aperea (cuis), entre otros (figura 3). El Cachapé V y Sotelo I indican una marcada selección y aprovechamiento del recurso coipo, 
especie con mayor representación en el registro de estos sitios. El carácter predecible y multifuncional de este recurso, ya que no sólo provee carne para la subsistencia sino también cuero y huesos que han sido transformados al sistema tecnológico (Santini y Plischuk, op. cit.), podría fundamentar su alta incidencia. Además no manifiesta estacionalidad, estando presente durante todo el año, y siendo una especie que no demandaría mayores esfuerzos para su captura (Dobrizhoffer, 1967 T II, González de Bonaveri, 2005, Acosta, 2005). Como complementariedad en la dieta, la pesca y recolección de moluscos ha tenido una significativa importancia. Los taxones más representados corresponden a: Liposarcus anisitsi (vieja del agua), Synbranchus marmoratus (anguila) y Lepidosiren paradoxa (pez pulmonado), todos ellos especies no migratorias, que estarían reflejando la existencia de un ambiente de tipo léntico (ambientes pertenecientes a cuerpos de aguas someros, rodeados de plantas y zonas de barro y lodo). Dentro de los moluscos de agua dulce encontramos una alta selección principalmente de Pomacea canaliculata chaquensis y en menor medida Bivalvos del género Diplodon.

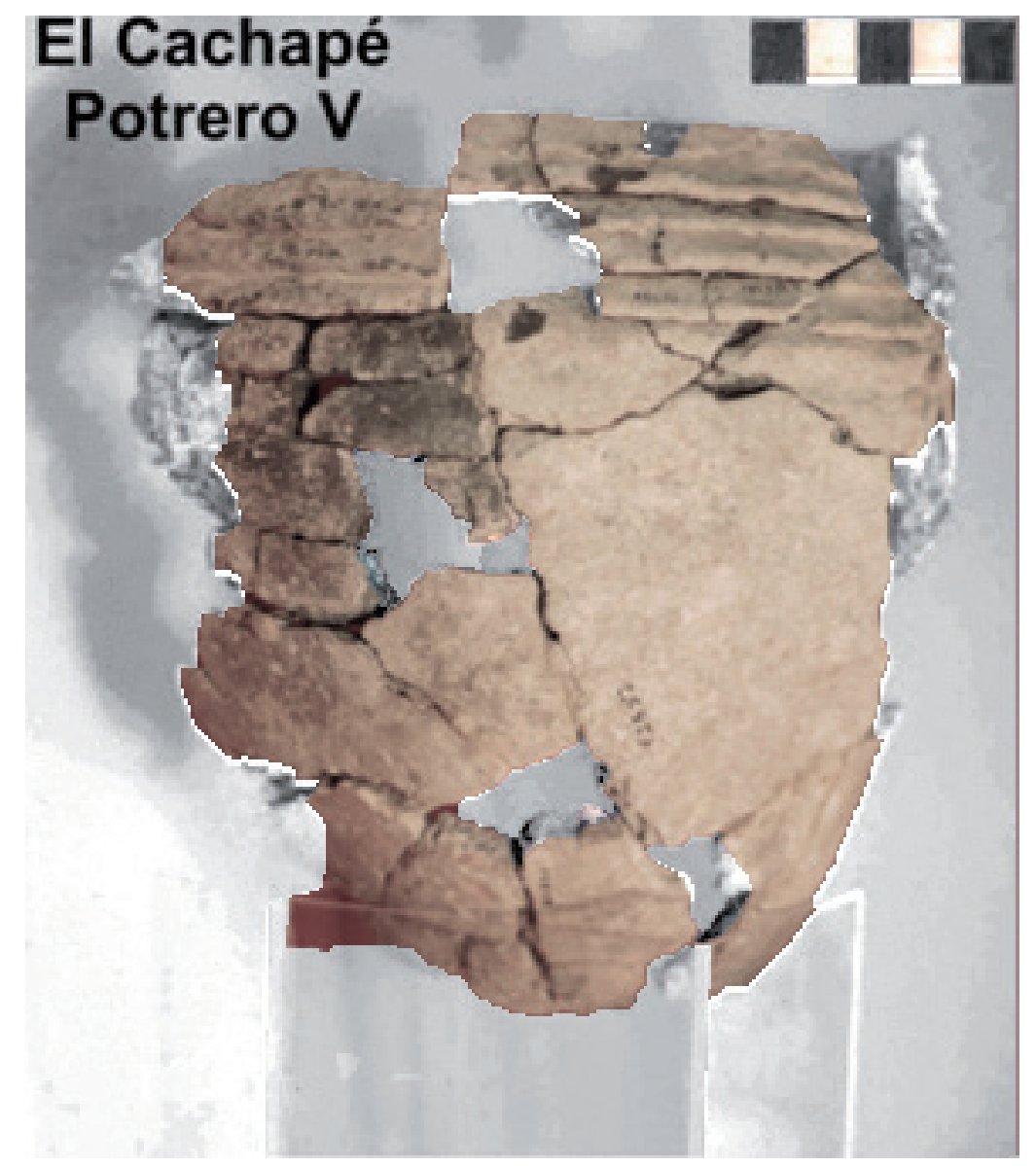

Foto 5. Material Cerámico característico de los sitios mencionados 
En cuanto a El Cachapé IVA y El Cachapé IVB la proporción de peces es más significativa que en los otros sitios con especies de mayor porte (Pimelodus albicans, Pimelodus sp., Pterodoras granulosus, entre otros), lo cual estaría reflejando un ambiente léntico, pero con un curso de agua de mayor caudal.

La dieta evidenciada en estos sitios es de tipo generalista y diversificada, representada por B. dichotomus y en menor medida peces, reptiles, roedores (Hydrochaeris hydrochaeris, M. coypus). Mientras que la recolección de moluscos no es significativa.

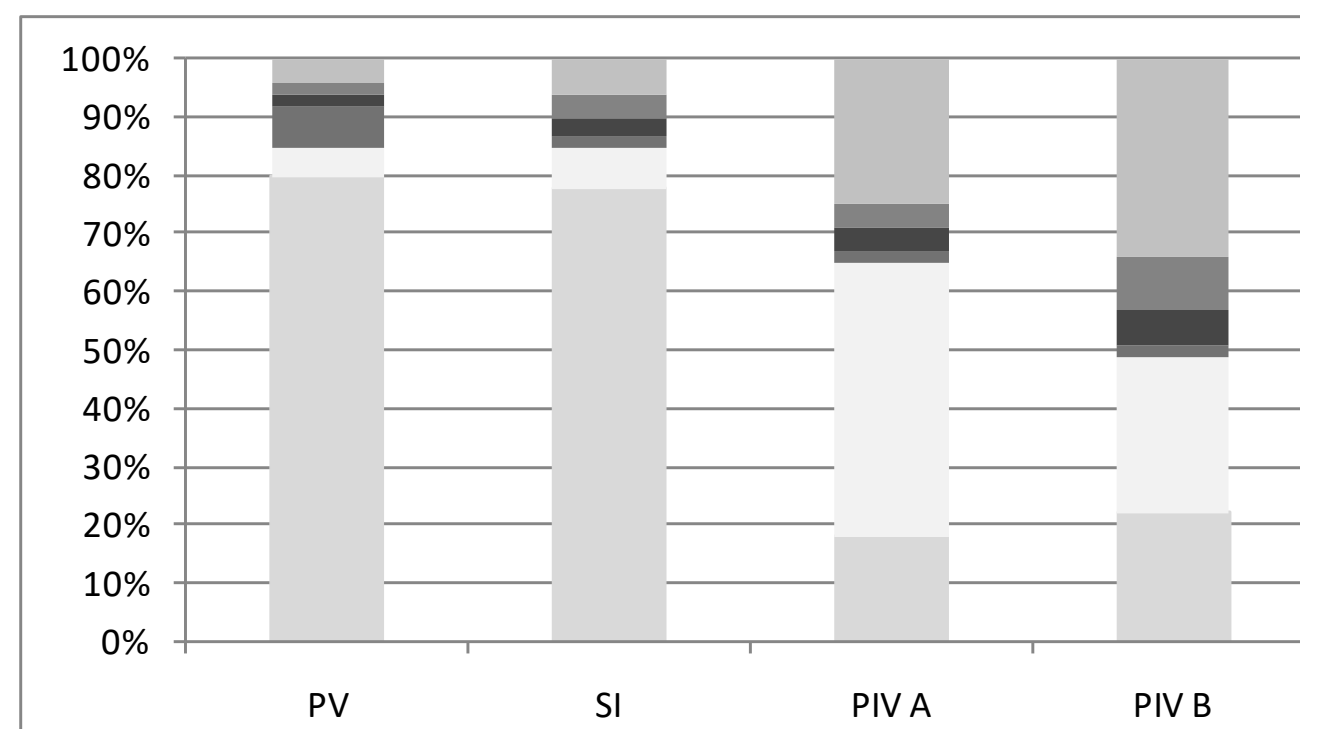

Myocastor corpus Peces Cavia aparea $\square$ Cervidos $\square$ Aves $\square$ Otros

Figura 3. NISP\% de los taxa mayormente representados en los conjuntos arqueofaunísticos.

Es de resaltar especialmente la presencia de restos óseos humanos en tres de los cinco sitios excavados (El Cachapé V, El Cachapé IVA, El Cachapé IVB). Se recuperaron restos de un individuo subadulto y dos adultos, dispuestos en forma directa en tierra dentro del espacio habitacional. También se encontraron restos humanos dispersos en relación a restos óseos faunísticos y material cerámico fragmentado, lo cual reviste particular importancia, ya sea para el estudio de los procesos de formación o bien para el análisis de procesamiento mortuorio secundario o como evidencia de violencia interpersonal. En El Cachapé V y El Cachapé IVB se recuperaron fragmentos de restos óseos humanos adultos, dispuestos en un contexto de basurero dentro del espacio habitacional en relación a restos óseos faunísticos y material cerámico fragmentado. En El Cachapé V se recuperaron restos óseos humanos (ambas rótulas, costilla, carpianos y metacarpianos) sobre el piso de ocupación y vinculados a restos de mamíferos, peces, moluscos bivalvos, gasterópodos de agua dulce y gasterópodos terrestres, próximo a estos restos, por debajo del piso 
de ocupación, se recuperó un enterratorio directo en tierra de un subadulto (Desántolo et al, 2005). En El Cachapé IVB se recuperaron fragmentos óseos humanos (frontal, epífisis proximal de tibia, epífisis distal de peroné, rótula, fragmentos de pelvis) entremezclados con los demás restos arquelógicos.

\section{Epílogo}

Todos los sitios arqueológicos se encuentran en zonas elevadas, estratégicamente emplazados, interconectando cursos de agua con bañados, controlando no sólo el acceso a los recursos propios de ambos cuerpos de agua, sino también a los primeros como posibles vías de comunicación (figura 4).

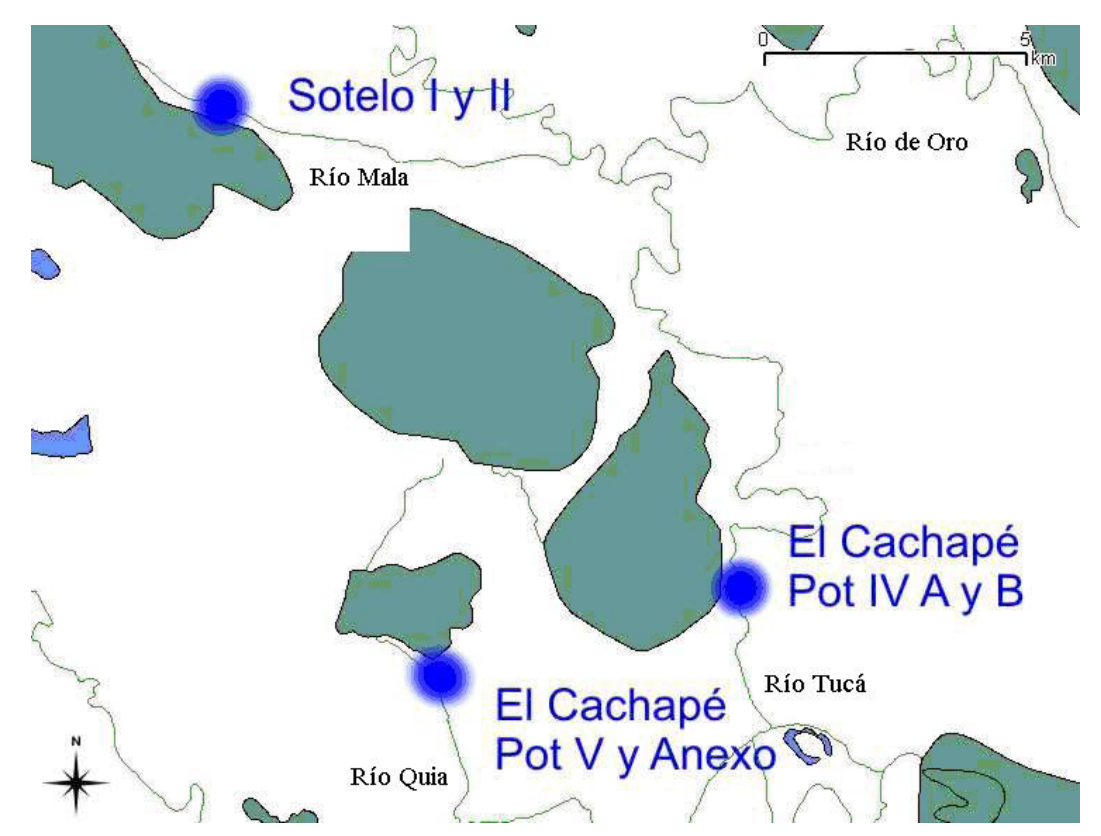

Figura 4. Ubicación de los sitios en relación a cursos de agua y bañados temporarios

Podemos ver que en el sector ribereño las áreas inundables, en las que observamos los montículos con actividades antrópicas, se elabora un espacio social de alto valor económico, donde los recursos predecibles durante todo el año demarcan esta condición. Además de estas características, la presencia de estructuras monticulares, las inhumaciones, adornos, estilo cerámico compartido, constituyen un paisaje social construido con presencia de individuos con roles y probables funciones diferenciadas dentro de la estructura familiar.

Hasta aquí es posible observar cómo las esferas del espacio físico y social se articulan, a las que debe sumarse el denominado espacio simbólico. Este, que va más allá del aspecto material, se configura como componente visible dentro de las unidades 
culturales a partir de compartir, por ejemplo (dentro de una gran variabilidad estilística) los mismos diseños y motivos decorativos en la cerámica (fundamentalmente el corrugado en su variante inciso), evidenciando un lenguaje, un ordenamiento propio que brinda información y al mismo tiempo demarca formas singulares que muestran la manera de comunicarse del grupo o grupos que la comparten. Este espacio simbólico es una parte fundamental en la construcción de la identidad grupal, y es un espacio regulado por dos ítems: su historicidad y control (Berger y Luckman, 1968).

Dada la naturaleza (cuali y cuantitativa) del dato que manejamos es posible inferir que el escenario regional en el cual se desarrollaron los procesos históricos fue construido socialmente, estableciéndose sus límites y territorialidad por las relaciones inter e intragrupales. Las diferencias y características particulares que se han presentado en otros grupos hacia el sur y hacia el oeste respaldan esta posición, y dan basamento inicial para caracterizar la dinámica étnica del sector en momentos prehispánicos.

Así, la construcción de un espacio propio y el mantenimiento de ciertas categorías que identifican al grupo en interacción con otros, como la presencia de aspectos particulares de la cerámica indicativos de funcionalidad, tecnología y caracterización estilística, respaldan estas ideas.

Ello no sólo se refleja en lo arqueológico, sino también en las numerosas crónicas, trabajos etnohistóricos y etnográficos en los cuales podemos observar distintas etnias ocupando espacios similares pero con la elaboración de un paisaje totalmente distinto (v.g. Toba/Wichí). Del análisis de la información disponible en su conjunto, especial dimensión y significación tiene el abundante dato etnohistórico y etnográfico toda vez que, desde su consideración, pueden construirse panoramas más integradores, válidos para la interpretación del pasado. El relato de los antiguos pobladores del Gran Chaco frecuentemente alude a personas actuales o históricas a quienes se les atribuyen el poder político sobre espacios territoriales amplios y numerosos grupos locales. (Braunstein, 2008). En cambio, las relaciones entre los grupos que estaban separados físicamente se establecían mediante un complejo sistema de preeminencias y atributos simbólicos que definían el vínculo entre los representantes de los grupos locales. Estas relaciones podrían calificarse como "políticas“ y regulaban la trama más o menos estable de las alianzas. El marco ritual de ese sistema institucional fue el de las antiguas ceremonias de bebida y los trofeos de guerra; en particular los escalpos de los enemigos muertos que constituían los principales atributos simbólicos puestos en juego en cada contexto cosmovisional para la fijación de las jerarquías (Braunstein, op.cit).

Ese entramado social con un ámbito de poder definido, ese paisaje construido y mantenido por los grupos ribereños prehispánicos en interacción con otros grupos, está en proceso a ser develado. A ello apuntamos con, éste, nuestro criterio.

\section{Referencias Bibliográficas}

Arellano López J. 2009. Culturas prehispánicas del Napo y el Aguarico. Amazonia Ecuatoriana. Ed. Centro Cultural José Pío Aza. Lima, Perú. 
Balbarrey G.; Calandra H.; Couso G.; Lamenza G.; Aguirre M. B. 2003. Nuevos aportes al análisis cerámico del sector central del Gran Chaco Argentino. En: XXIII Encuentro de Geohistoria Regional (IIGHI-CONICET).

Barnard A. 1983. Territoriality among human foragers: Ecological models and an application to four bushman groups. Current Anthropology, núm. 24 (1), pp. 47-66 [comments and reply].

Berger, P. y Luckmann, T. 1968. La Construcción Social de la Realidad. Ed. Amorrortu. Argentina. Calandra H. A., Méndez M. G., Salceda S. A., Lamenza G., Lanciotti M., Del Papa L., Duhalde N. 2000. Estudio preliminar de los restos cerámicos hallados en el sitio arqueológico "El Cachapé" (Chaco, Argentina). En: Actas del XX Encuentro de Geohistoria Regional. Vol I: 157-171.

Calandra H. A., Balbarrey G., Couso G., Lamenza G., Aguirre B., Duhalde N. 2004. El Sitio Las Bolivianas (Formosa): análisis comparativo del material cerámico del Sector Central del Gran Chaco argentino. En: XXIV Encuentro de Geohistoria Regional (IIGHI-CONICET).

Calandra H. A., Santini M., Salceda S., Lamenza G. 2004. Arqueología ribereña del Chaco: Presentación de un nuevo sitio arqueológico. En: XXIV Encuentro de Geohistoria Regional (IIGHI-CONICET).

Cardoso de Oliveira R. 1976. Identidad, Etnia e Estructura Social. Universidad Federal de Brasilia, Sao Paulo, Livraria Pioneira Editorial, p.4.

Cashdan, E. 1983. Territoriality among human foragers: Ecological models and an application to four bushman groups. Current Anthropology, núm. 24 (1), pp. 47-66.

Colazo S., Méndez M.G., Calandra H.A., Ferrarini S.O., Salceda S.A. 2002. Estudio preliminar del sitio arqueológico "El Chancho", Departamento de San Fernando. Provincia del Chaco. En: Actas del XXI Encuentro de Geohistoria Regional. Pp 68-74.

Criado Boado, F. 1999. Del Terreno al espacio: Planteamientos y Prespectivas para la Arqueología del Paisaje. En: CAPA (Criterios e Convencións en Arqueoloxía da Paisaxe) 6, Santiago de Compostela.

De Feo, C., H.A. Calandra., M. Santini., B. Aguirre., G. Lamenza., M.I. Lanciotti., L. Del Papa y A. Porterie. 2002. Localización espacial y caracterización cultural de sitios arqueológicos del Gran Chaco Meridional. En: XXII Encuentro de Geohistoria Regional. 263-294 pp. Chaco, Argentina.

Desántolo, B; Santini, M; Salceda, S. 2005. Arqueología Chaqueña 4: hallazgo de restos humanos en el sitio arqueológico "El Cachapé - Potrero V". Informe preliminar. En: Actas del XXV Encuentro de Geohistoria Regional. IIGHI-CONICET. Corrientes.

Dyson-Hudson, R. y Smith, E. A. 1978. Human Territoriality: An Ecological Reassessment. American Anthropologist, núm. 80 (1), pp. 21-41.

Eremites de Oliveira, J. 2004. Arqueología das Sociedades Indígenas no Pantanal. Campo Grande, MS: Ed. Oeste. Brasil

Ginzburg, R y J. Adámoli. 2006. Situación Ambiental en el Chaco Húmedo. En Brown, A., U. Martinez Ortiz, M. Acerbi y J. Corcuera (Eds.), La Situación Ambiental Argentina 2005, Fundación Vida Silvestre Argentina, Buenos Aires, pp 103-113.

Ingold, T. 1986. Territoriality and Tenure: the appropriation of space in hunting and gathering societies. In T. Ingold The Appropriation of Nature: Essays on Human Ecology and Social Relations . Manchester: University of Manchester Press.

Richard Daly. 1999. On the social relations of the hunter-gatherer band. En: The Cambridge Encyclopedia of Hunter and Gatherers. Richard B. Lee and Richard Daly (Eds.) Cambridge University Press. pp. 399-410. 
Lamenza, G.; Aguirre, B.; Calandra, H. 2005. Alfarería arqueológica del sector Paraguay-Paraná del Chaco Meridional: su sistematización e identidad. En: Actas del XXV Encuentro de Geohistoria Regional. IIGHI-CONICET. Corrientes.

Lamenza G., Balbarrey G., Aguirre B., Calandra H. 2006. Complejidad e interacción de sociedades prehispánicas ribereñas del Gran Chaco argentino. En Actas del XXV Encuentro de Geohistoria Regional. IIGHI-CONICET. Publicación en CD formato libro. Resistencia, Chaco.

Lamenza G., Santini M., Castro J. 2007. Ocupación del espacio en el Sector Ribereño ParaguayParaná (Chaco-Argentina). En Actas del $2^{\circ}$ Encuentro de Discusión Arqueológica del Nordeste Argentino. Paraná, Entre Ríos.

Neiff. 1986. Sinópsis ecológica y estado actual del Chaco Oriental. Ambiente Subtropical, 1: 5-35.

Morello, J. 1968. La vegetación de la República Argentina. Las grandes unidades de vegetación y ambiente del Chaco argentino". INTA (Serie Fitogeográfica N¹0). Buenos Aires.

Pauketat, T. 2001. Practice and History in Archaeology: An Emerging Paradigm. Anthropological Theory 1:73-98.

Salceda S.A., Méndez M.G., Calandra H.A., Santini M., Giovannetti M.A., Couso G., 2000. Análisis preliminar de los restos faunísticos del sitio arqueológico "El Cachapé" (Chaco, Argentina). En Actas del XX Encuentro de Geohistoria Regional, Vol II: 795-806.

Santini M., Salceda S.A., De Santis L., Del Papa L., 2003. Primeras aproximaciones a estudios tafonómicos en sitios de la región ribereña-paranaense del Chaco meridional. En Actas del XXIII Encuentro de Geohistoria Regional. Pp. 364-369.

Santini M. y Plischuk M. 2006. Subregión Ribereña Paraguay Paraná: análisis de los conjuntos de artefactos óseos provenientes de 2 sitios arqueológicos. En Actas del XXV Encuentro de Geohistoria Regional. IIGHI-CONICET. Publicación en CD formato libro. Resistencia, Chaco

Balbarrey G.; Calandra H.; Couso G.; Lamenza G.; Aguirre M. B. 2003. Nuevos aportes al análisis cerámico del sector central del Gran Chaco Argentino. En: XXIII Encuentro de Geohistoria Regional (IIGHI-CONICET).

Calandra H. A, Salceda S. A., González O., Cid de la Paz M., Caló M. 2003. Arqueología Chaqueña 3: nuevas evidencias de cordelería impresa. En Actas del XXII Encuentro de Geohistoria Regional. Pp. 72-76. ISBN 950-692-060-5.

Calandra H. A., Santini M., Salceda S., Lamenza G. 2004. Arqueología ribereña del Chaco: Presentación de un nuevo sitio arqueológico. En : XXIV Encuentro de Geohistoria Regional (IIGHI-CONICET).

Calandra H. A., Balbarrey G., Couso G., Lamenza G., Aguirre B., Duhalde N. 2004. El Sitio Las Bolivianas (Formosa): análisis comparativo del material cerámico del Sector Central del Gran Chaco argentino. En: XXIV Encuentro de Geohistoria Regional (IIGHI-CONICET).

Colazo S., Méndez M.G., Calandra H.A., Ferrarini S.O., Salceda S.A. 2002. Estudio preliminar del sitio arqueológico "El Chancho", Departamento de San Fernando. Provincia del Chaco. En Actas del XXI Encuentro de Geohistoria Regional. Pp 68-74.

Rogge, J. H. 1996. A Tradição Pantanal: Uma Nova Tradição Cerâmica nas Terras Baixas SulAmericanas. En: Simposio Arqueología de Las Tierras Bajas, Montevideo.

Schmitz, P. I. y Beber, M. V. 1996. Aterros no Pantanal do Mato Grosso do Sul. En: Simposio Arqueología de Las Tierras Bajas, Montevideo.

Schmitz, P. I.; Rogge, J. H.; Rosa, A. O.; Beber, M. V. 1998. Aterros Indígenas no Pantanal do Mato Grosso do Sul. Pesquisas/Antropologia, São Leopoldo, № 54. 